\title{
Three-Dimensional Histologic Validation of High-Resolution SPECT of Antibody Distributions Within Xenografts
}

\author{
Woutjan Branderhorst ${ }^{1-3}$, Erwin L.A. Blezer ${ }^{1}$, Mischa Houtkamp ${ }^{4}$, Ruud M. Ramakers ${ }^{2,5,6}$, Jeroen H. van den Brakel ${ }^{4}$, \\ Henry Witteveen ${ }^{4}$, Frans van der Have ${ }^{5,6}$, Hugo A. Gratama van Andel ${ }^{6}$, Brendan Vastenhouw ${ }^{5,6}$, Chao Wu ${ }^{5,6}$, \\ Marijke Stigter-van Walsum ${ }^{7}$, Guus A.M.S. van Dongen ${ }^{7}$, Max A. Viergever ${ }^{1}$, Wim K. Bleeker ${ }^{4}$, and Freek J. Beekman ${ }^{5,6}$ \\ ${ }^{I}$ Image Sciences Institute, University Medical Center Utrecht, Utrecht, The Netherlands; ${ }^{2}$ Rudolf Magnus Institute of Neuroscience, \\ University Medical Center Utrecht, Utrecht, The Netherlands; ${ }^{3}$ Department of Biomedical Engineering and Physics, Academic \\ Medical Center Amsterdam, Amsterdam, The Netherlands; ${ }^{4}$ Genmab B.V., Utrecht, The Netherlands; ${ }^{5}$ Section Radiation, Detection, \\ and Medical Imaging, Applied Sciences, Delft University of Technology, Delft, The Netherlands; ${ }^{6}$ MILabs B.V., Utrecht, The \\ Netherlands; and ${ }^{7}$ Department of Nuclear Medicine and PET Research, VU University Medical Center, Amsterdam, The Netherlands
}

Longitudinal imaging of intratumoral distributions of antibodies in vivo in mouse cancer models is of great importance for developing cancer therapies. In this study, multipinhole SPECT with sub-halfmillimeter resolution was tested for exploring intratumoral distributions of radiolabeled antibodies directed toward the epidermal growth factor receptor (EGFr) and compared with full 3-dimensional target expression assessed by immunohistochemistry. Methods: ${ }^{111}$ In-labeled zalutumumab, a human monoclonal human EGFr-targeting antibody, was administered at a nonsaturating dose to 3 mice with xenografted A431 tumors exhibiting high EGFr expression. Total-body and focused in vivo tumor SPECT was performed at 0 and $48 \mathrm{~h}$ after injection and compared both visually and quantitatively with full 3-dimensional immunohistochemical staining for EGFr target expression. Results: SPECT at $48 \mathrm{~h}$ after injection showed that activity was predominantly concentrated in the tumor $(10.5 \% \pm 1.3 \%$ of the total-body activity; average concentration, $30.1 \% \pm 4.6 \%$ of the injected dose per cubic centimeter). ${ }^{111} \mathrm{In}$-labeled EGFr-targeting antibodies were distributed heterogeneously throughout the tumor. Some hot spots were observed near the tumor rim. Immunohistochemistry indicated that the antibody distributions obtained by SPECT were morphologically similar to those obtained for ex vivo EGFr target expression. Regions showing low SPECT activity were necrotic or virtually negative for EGFr target expression. A good correlation $(r=0.86, P<0.0001)$ was found between the percentage of regions showing low activity on SPECT and the percentage of necrotic tissue on immunohistochemistry. Conclusion: Multipinhole SPECT enables high-resolution visualization and quantification of the heterogeneity of ${ }^{111}$ In-zalutumumab concentrations in vivo.

Key Words: micro-SPECT; histology; antibody; zalutumumab; EGFr

J Nucl Med 2014; 55:830-837

DOI: 10.2967/jnumed.113.125401

$\mathbf{T}$

he development of molecular imaging opens up exciting opportunities for advancing the detection, diagnosis, and treatment

\footnotetext{
Received May 1, 2013; revision accepted Jan. 27, 2014.

For correspondence or reprints contact: Woutjan Branderhorst, Department of Biomedical Engineering and Physics, Academic Medical Center Amsterdam, Meibergdreef 9, 1105AZ Amsterdam, The Netherlands. Published online Mar. 31, 2014.

COPYRIGHT (c) 2014 by the Society of Nuclear Medicine and Molecular Imaging, Inc.
}

of cancer (1). In recent years, there has been considerable interest in the development and imaging of monoclonal antibody therapies $(2,3)$. A significant amount of research is aimed at achieving targeted anticancer drug delivery by conjugating monoclonal antibodies to highly toxic agents $(4,5)$.

Zalutumumab (6) is a human IgG1 monoclonal antibody, specific for the human epidermal growth factor receptor (EGFr), that has been developed for cancer treatment. EGFr is overexpressed in a variety of cancers and is associated with a more aggressive malignant phenotype and poor prognosis (7). Zalutumumab has 2 mechanisms of action that result in killing of EGFr-positive tumor cells. Aside from the blockade of ligand binding, there is also induction of antibody-dependent cell-mediated cytotoxicity, which is typical for IgG1 monoclonal antibodies (8). The efficacy of zalutumumab on binding to EGFr has been shown both in vitro and in vivo (9), but limited information is available on tumor penetration.

PET has been successfully applied to image the typically heterogeneous intratumoral distribution in human solid tumors (10). Although the spatial resolution of PET has recently been advanced into the submillimeter range, which allows similar imaging studies in mice (11), small-animal SPECT provides even higher (less than half-millimeter) spatial resolution, which can be achieved by exploiting focused multipinhole SPECT designs (12). In addition, most SPECT labels have a longer half-life and SPECT uses sets of tracers different from (and often complementary to) those available for PET. As a result, for many applications small-animal SPECT is chosen as the preferred method to image intratumoral immunoconjugate distributions in small xenografts in mice. Various studies have demonstrated that small-animal SPECT results in highly quantitative images (13-15).

Many SPECT studies on the relationship between radiolabeled antibody uptake and target receptor expression in a mouse xenograft model assume that these constructs are proportionally related $(16,17)$. However, experiments aimed at validating this assumption against histology have been limited to comparisons involving 2-dimensional (2D) autoradiography $(16,18)$ or comparisons with only a single histology slice (18-21). To our knowledge, no work has yet reported a slice-by-slice comparison of antibody uptake by xenografts in entire SPECT volumes against 3-dimensional (3D) histology.

The imaging capabilities of small-animal SPECT have been improved dramatically over the last few years (22). The aim of this study was to do an in-depth validation of the use of SPECT with the highest available resolution (less than half-millimeter) for $3 \mathrm{D}$ 
imaging of heterogeneous tumor uptake of ${ }^{111}$ In-labeled zalutumumab by comparing it with the ex vivo EGFr expression distribution as obtained by full 3D immunohistochemistry. To provide such a thorough 3D comparison, stacks of histology sections were constructed using dedicated protocols.

\section{MATERIALS AND METHODS}

\section{Antibody Generation and Labeling with ${ }^{111}$ In}

A human monoclonal antibody targeting human EGFr (zalutumumab, monoclonal antibody 2F8; Genmab) was generated as described previously (23). For labeling of zalutumumab with ${ }^{111} \mathrm{In}$, the antibody was conjugated with p-benzyl isothiocyanate-1,4,7,10-tetraazacyclododecane-1,4,7,10-tetraacetic acid (p-SCN-Bz-DOTA), as described previously (24). In short, a buffer exchange of the antibody to $0.1 \mathrm{M} \mathrm{NaHCO}_{3}$ ( $\mathrm{pH} 9$ ) was performed. Antibody (6 mg) was incubated with $0.2 \mathrm{mg}$ of DOTA, and the premodified antibody was purified using gel filtration. The antibody-DOTA complex ( $3 \mathrm{mg}$ ) was labeled with $750 \mathrm{MBq}$ of ${ }^{111} \mathrm{In}$ ( $30 \mathrm{~min}$ at $45^{\circ} \mathrm{C}$ ). After labeling, the mixture was incubated for $5 \mathrm{~min}$ with ethylenediamine tetraacetic acid, $0.05 \mathrm{~mol} / \mathrm{L}$, and the ${ }^{111} \mathrm{In}-\mathrm{p}-\mathrm{SCN}-$ Bz-DOTA-zalutumumab was purified using gel filtration. For quality control, instant thin-layer chromatography and cell binding assays were performed as described previously (24). Instant thin-layer chromatography analyses revealed a radiochemical purity of $99.9 \%$ for the labeled antibody. The immunoreactive fraction was $88 \%$.

\section{Mouse Xenograft Tumor Model}

All animal procedures were approved by the Utrecht University animal ethics committee and were performed in accordance with international guidelines on handling laboratory animals. A wellcharacterized subcutaneous xenograft tumor model was used (25). Briefly, 8- to 12-wk-old female severe combined immunodeficiency mice (C.B-17/IcrPrkdc-scid/CRL, $n=3$ ) were purchased from Charles River Laboratories and housed in the Central Laboratory Animal Facility in a sterile filter-top cage with water and food provided ad libitum. Throughout the experiment, the mice were checked at least twice a week for signs of discomfort. A431 cells (an EGFr-overexpressing human epidermoid cancer cell line) were obtained from the Deutsche Sammlung von Mikroorganismen und Zellkulturen (cell line number, ACC 91). The cells were cultured in RPMI 1640 medium (BioWhittaker) supplemented with heat-inactivated fetal calf serum (10\%; Wisend), penicillin $(50 \mathrm{IU} / \mathrm{mL})$, and streptomycin $(50 \mu \mathrm{g} / \mathrm{mL})$. Cells were detached by using trypsin-ethylenediamine tetraacetic acid in phosphatebuffered saline, harvested in the log phase (confluence, $\sim 70 \%$ ), and tested for EGFr expression and potential mycoplasm contamination before the experiment. Five million tumor cells were inoculated subcutaneously into the right flanks of each of the 3 mice after removal of hair, using a 29-gauge needle. The tumors were measured by Vernier calipers, and volumes were estimated as $0.52 \times$ length $\times$ width $^{2}$ (in $\mathrm{mm}^{3}$ ) (26). On the day of the first SPECT imaging session, the estimated tumor volumes were $300 \mathrm{~mm}^{3}, 290 \mathrm{~mm}^{3}$, and $330 \mathrm{~mm}^{3}$, and the associated mice had body weights of $22 \mathrm{~g}, 22 \mathrm{~g}$, and $24 \mathrm{~g}$, respectively.

\section{Ultra-High-Resolution SPECT}

SPECT was performed with the U-SPECT-II system (MILabs). This device uses stationary detectors with exchangeable collimators for different-sized animals or for specific organs (12). In this study, targeted SPECT acquisitions were performed (27) using a mouse collimator with 75 pinholes (diameter, $0.6 \mathrm{~mm}$ ).

\section{Imaging Protocols}

Nineteen days after tumor cell inoculation, the mice were anesthetized using isoflurane (1\%-2\% in air) and a nontherapeutic effective $5 \mathrm{mg} / \mathrm{kg}$ dose of ${ }^{111} \mathrm{In}$-labeled zalutumumab $(29.6 \pm 1.6$ $\mathrm{MBq}$ ) was injected into the tail vein. Immediately after injection, a 45-min in vivo total-body SPECT/CT scan was obtained while anesthesia was maintained. Body temperature, heart rate, and breathing frequency were monitored continuously and kept at normal physiologic values. At $48 \mathrm{~h}$ after injection, anesthesia was reinitiated and a $45-\mathrm{min}$ in vivo focused SPECT scan of the tumor was obtained, followed by a second 45-min total-body SPECT/CT scan. Subsequently, the mice were euthanized by anesthetic overdose. Immediately after each last scan, the entire mouse carcass was snap-frozen by immersion in $-80^{\circ} \mathrm{C}$ isopentane. To avoid tissue repositioning and deformation, the carcasses were immersed while still situated on the animal bed. The activity in the mouse carcasses was measured using a dose calibrator, and the carcasses were stored in a $-80^{\circ} \mathrm{C}$ freezer until further use. The total-body and focused tumor SPECT images were reconstructed using pixel-based ordered-subset expectation maximization with 6 iterations and 16 subsets (28), on 0.375 -mm (total-body) and 0.1875 -mm (focused tumor) isotropic $3 \mathrm{D}$ voxel grids. To increase image contrast, scatter correction was applied similarly to a triple-energy-window-based correction described earlier (14). Briefly, 2 photopeak energy windows (150-185 and $220-270 \mathrm{keV}$ ) corresponding to the 2 photopeaks of ${ }^{111}$ In were selected, as well as 3 background energy windows (133-150, 189-218, and 275$292 \mathrm{keV}$ ). The CT scans were reconstructed using cone-beam filtered backprojection (Feldkamp algorithm) on an $83-\mu \mathrm{m}$ voxel grid. The focused tumor SPECT images were corrected for nonuniform attenuation by a method described earlier (14), using CT images.

\section{Full-Tumor Tissue Sectioning}

After the radioactivity had sufficiently decayed, the tumors were removed from the carcass, including a small margin of surrounding tissue. A cryostat (Microm HM560; Thermo Fisher Scientific) was used to obtain $10-\mu \mathrm{m}$-thick transaxial tissue sections from the resulting tissue block. Sections were obtained at approximately $0.375-\mathrm{mm}$ intervals. At the beginning of each interval, a digital photograph of the tissue block face was acquired to capture the shape of the tissue before any deformations resulting from the sectioning and subsequent immunohistochemical staining. The camera (EOS 550D with EF $100 \mathrm{~mm} \mathrm{f} / 2.8$ macro lens [Canon] and a Teleplus PRO 300 DG [Kenko]) was mounted onto a tripod standing on the floor close to the cryostat. Severely deformed sections were discarded and replaced by an adjacent section. Furthermore, some additional adjacent sections were obtained at each location for additional stainings. All eventually used sections were at a maximum physical distance of $40 \mu \mathrm{m}$ from the start of the interval at which the corresponding photograph was taken. The morphologic changes over this short distance were negligible.

\section{Immunohistochemistry}

The tissue sections $(10 \mu \mathrm{m})$ were fixed in acetone $(10 \mathrm{~min}$, at room temperature), and endogenous peroxidase was blocked by citrate/ phosphate buffer ( $\mathrm{pH} 5.8$ ). Aspecific binding to Fc receptors was blocked with human normal serum. Tissue sections were immunostained for EGFr target expression by ex vivo preincubation with zalutumumab $(1 \mu \mathrm{g} / \mathrm{mL}$, $1 \mathrm{~h}$, room temperature). Ex vivo and in vivo administered zalutumumab was detected with polyclonal rabbit-antihuman $\operatorname{IgG}(\kappa)(\mathrm{DAKO})$ at a concentration of $1 \mu \mathrm{g} / \mathrm{mL}$ (30 min, room temperature). The rabbit antihuman antibodies were subsequently detected with a horseradish peroxidase conjugated goat antirabbit IgG dextran-polymer (Gt-anti-RbIgG-Powervisionhorseradish peroxidase; Immunologic). Mouse macrophages were immunostained with rat-antimouse macrophage monoclonal antibody (clone $\mathrm{F} 4 / 80$ ) at $10 \mu \mathrm{g} / \mathrm{mL}$ ( $1 \mathrm{~h}$, room temperature), and subsequent incubations were performed with rabbit-antirat-IgG and Gt-anti-RbIgGPowervision-horseradish peroxidase (Immunologic). The presence of horseradish peroxidase was visualized by adding 3,3'-diaminobenzidine (Sigma-Aldrich) development solution, resulting in brown staining, or by adding amino-ethyl-carbazole development solution, resulting in red staining. Sections were counterstained with hematoxylin (blue nuclear 
staining), and a coverslip in glycerogel was applied. All immunostained tissue sections were scanned using the Ariol SL-50 system (Leica Microsystems) at low magnification $(\times 4)$ and at high magnification $(\times 20)$ for detailed analysis, using the software module "GenSight visual only."

\section{Construction of 3D Histology Stack}

For each tumor, a 3D stack of stained histologic sections was created by first stacking the block-face photographs and then registering each histologic section to its corresponding image in the stack. The stacked block-face images were first aligned by registering each $2 \mathrm{D}$ image to its adjacent image using StackReg (29) (version March 2, 2005/TurboReg, version June 19,2008) allowing only rigid in-plane transformations and in-plane isotropic scaling. In the resulting stack, 4 markers that were fixed with respect to the tissue mounting table were aligned within a maximum deviation of 1 pixel (size, $0.043-0.050 \mathrm{~mm}$ isotropic; mean \pm $\mathrm{SD}, 0.046 \pm 0.004 \mathrm{~mm}$ ). All further processing of the block-face photographs as well as the digitized histology images was performed on the intensities in the blue color channel, which showed the largest contrast between various tumor structures for both image types. Each of the digitized stained histology images was registered to its corresponding photograph in the 3D block-face stack by means of Elastix (30) (version 4.5) using an iterative gradient-descent algorithm. An affine transformation model was used for the registration, since the main deformation encountered in the sections was a compression of the tissue along the cutting direction and severely torn or folded sections were discarded during the sectioning. The registration was performed by first manually defining a set of corresponding landmark points at the boundaries of clearly distinguishable structures within the tumor, such as shown in Figures $1 \mathrm{~A}$ and $1 \mathrm{~B}$, and then performing an automatic registration with the similarity measure set to a weighted combination of the Euclidian distance between these points (weight, 0.25) and mutual information between the original images (weight, 0.75). Mutual information was calculated only over the image pixels that contained xenograft tissue, as defined by tumor masks that were manually delineated for each $2 \mathrm{D}$ block-face image (Fig. 1C). The resulting registered stained histology images (an example registration is displayed in Fig. 1D) were each assigned a thickness of $0.375 \mathrm{~mm}$ and stacked to create the 3D EGFrstained histology image volumes. Some histology images at the edges of the tissue block were discarded because of a lack of corresponding structures for registration. As a result, the number of usable histology sections was slightly lower than the number of SPECT slices encompassing the tumor $(24,29$, and 23 usable consecutive sections versus 28,30 , and 27 slices, respectively).

\section{Registering SPECT to Histology}

The in vivo SPECT image was registered directly to the stained histology stack, without any reference to the block-face photographs used to create the stack. First, the SPECT image was resampled to the voxel grid of the histology stack using trilinear interpolation. Then, the tumor tissue masks that were defined on the 2D block-face images (Fig. 1C) were used to set all nontumor voxels in the histology stack to zero. Finally, the SPECT image was registered to the resulting masked volume by maximizing mutual information by applying Elastix (30), using an iterative gradient-descent algorithm and allowing only rigid 3D transformations.

\section{Image Visualization and Analysis}

The total-body and focused SPECT volumes were smoothed using 3D isotropic gaussian postfilters with full widths at half maximum of, respectively, 1.0 and $0.4 \mathrm{~mm}$. To determine the percentage injected dose (\%ID) taken up by the tumor and the total body, corresponding $3 \mathrm{D}$ regions of interest were drawn manually and their reconstructed voxel intensity sums were related to dose calibrator measurements. Maximum-intensity projections of the total-body SPECT/CT volumes
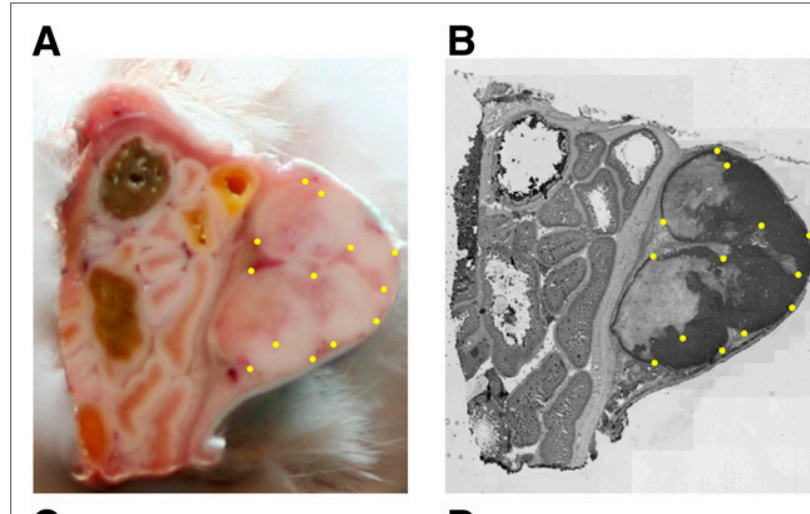

C

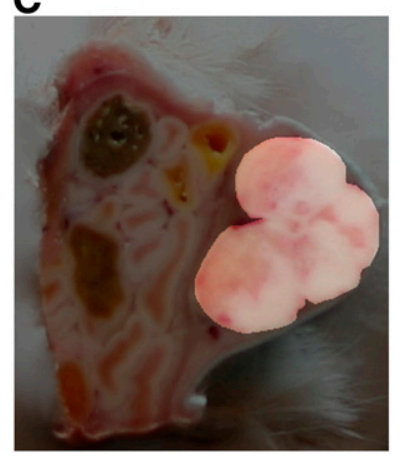

D

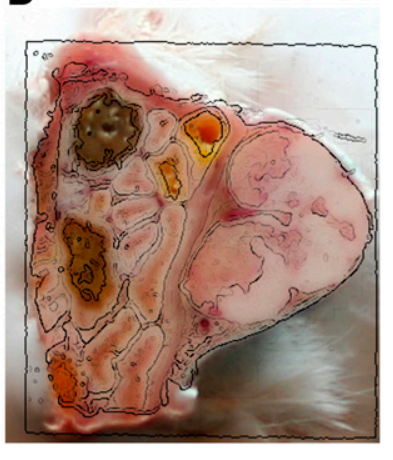

FIGURE 1. Example in-plane 2D registration of EGFr-stained histology image to corresponding tissue block photograph. (A) Block-face image with yellow dots denoting manually selected landmarks. (B) Blue color channel extracted from original RGB color histology image, with yellow dots denoting points corresponding to landmarks in A and EGFr target expression in dark gray. (C) Tumor mask used during registration and calculation of percentage necrosis. (D) Edges of registered histology image (detected using Canny edge detector) overlaid onto block-face photograph.

were generated using PMOD (PMOD Technologies). The consecutive histology sections were fused with 0.375 -mm-thick slices from the registered focused SPECT volume and compared visually.

To further characterize the agreement between the SPECT and immunohistochemistry image content, we compared slice by slice the percentage of low-activity pixels on SPECT with the percentage of necrotic tissue on immunohistochemistry. The percentage of necrotic tissue was measured by manually segmenting the necrotic regions in the registered full-color EGFr images and dividing the total necrotic area by the area of the corresponding tumor mask (Fig. 1C). To avoid mismatches due to misregistration of the relatively small slices at the beginning and end of each stack, all slices having $0 \%$ necrosis were excluded. Low-activity pixels in the SPECT slices were classified by thresholding the reconstructed activity. The resulting low-activity areas divided by the tumor mask areas were then compared with the corresponding fractions of necrotic tissue by calculating the Pearson correlation coefficient for the set of all slices from the 3 mice together. To quantify and visualize the best agreement between SPECT and immunohistochemistry that could be found by thresholding alone, this procedure was repeated for a range of threshold values. Each threshold was absolute and applied to the entire set of all slices from all 3 mice, resulting in 1 correlation coefficient for each threshold. The threshold resulting in the highest correlation coefficient was analyzed for robustness to variation of the threshold. Subsequently, a scatterplot was generated from the data with the highest coefficient, and least squares linear regression was performed. 


\section{RESULTS}

\section{Total-Body Images}

Figure 2 compares the total-body SPECT/CT image obtained from 1 of the 3 mice within $1 \mathrm{~h}$ after administration of ${ }^{111}$ In-labeled zalutumumab $(\mathrm{t}=0 \mathrm{~h})$ with the corresponding total-body image obtained $48 \mathrm{~h}$ later $(\mathrm{t}=48 \mathrm{~h})$. At $\mathrm{t}=0 \mathrm{~h}$, the activity was present mainly in the blood vessels, the heart, the bladder, and the injection site. This finding was also observed in the other 2 mice. At $t=48 \mathrm{~h}$, apart from some accumulation in the snout and ears (not encountered in the other 2 mice), the activity was mainly in the tumor. These 2 images demonstrate the anticipated process of specific on-target (EGFr) binding over time.

Region-of-interest analysis revealed that at $\mathrm{t}=48 \mathrm{~h}$ the tumor contained $9.0 \%-11.4 \%$ (mean, $10.5 \% \pm 1.3 \%$ ) of the total-body activity. Using dose calibrator measurements of the mouse carcasses, we determined that the corresponding absolute activities were 1.40-1.72 MBq (mean, $1.59 \pm 0.17 \mathrm{MBq}$ ). Correcting for radioactive decay, this is equivalent to $2.35-2.94 \mathrm{MBq}$ (mean, $2.71 \pm 0.32 \mathrm{MBq}$ ) at the time of injection, constituting 8.2-10.0 $\% \mathrm{ID}$ (mean, $9.2 \pm 0.9 \% \mathrm{ID}$ ), or $24.8-33.3 \% \mathrm{ID} / \mathrm{cm}^{3}$ (mean, $30.1 \pm$ $\left.4.6 \% \mathrm{ID} / \mathrm{cm}^{3}\right)$. The mouse shown in Figure 2 corresponds to the tumor shown in Figure 3C.

\section{Focused Tumor SPECT and Histology Images}

For each of the 3 mice, a set of equidistant SPECT tumor slices at $\mathrm{t}=48 \mathrm{~h}$ is displayed in Figure 3, together with corresponding images from the registered histology stacks. The high resolution of the SPECT images enables small substructures within the tumor to be distinguished. Activity was distributed heterogeneously throughout the tumor, exhibiting substantial internal regions containing almost no tracer and some small hot spots with relatively high concentrations. Supplemental Figures 1-3, containing similar sets of consecutive slices from all 3 tumors resliced in 3 orthogonal directions, allow further assessment of the correspondence in 3 dimensions (supplemental materials are available at http://jnm.snmjournals.org).

When the SPECT volume is compared with the EGFr-stained histology volume, many spatially corresponding morphologic details can be identified. In general, most regions of low uptake in the
SPECT slices were histologically characterized as necrotic tissue. Interestingly, the coregistration with the histology stack reveals that some spots exhibiting a relatively high ${ }^{111}$ In concentration on SPECT, such as the ones pointed out by the arrows in Figure 3, are located at the outer rim of the tumor or even outside the highly EGFr-expressing tumor region.

In Figure 4, the correspondence between the percentage of intratumoral low-activity pixels on SPECT and the percentage of necrotic tissue on immunohistochemistry is quantified (estimated concentration corresponding to the applied SPECT threshold, 4.5 $\mathrm{MBq} / \mathrm{cm}^{3}$ ). The scatterplot shows that a high and significant correlation coefficient $(r=0.86, P<0.0001)$ could be achieved between regions of low ${ }^{111}$ In uptake on SPECT and EGFr-negative areas on immunohistochemistry. This coefficient was stable with regard to the chosen threshold: within a margin of $20 \%$ (e.g., when raising or lowering the threshold value by $10 \%$ ) the lowest resulting $r$ was 0.85 , and within $30 \%$ the lowest $r$ was 0.83 . The regression line has a positive slope of 0.645 and a $y$-intercept close to the origin. When the point clouds of the individual mice are compared, it appears that the points have roughly the same shape as the point cloud from all mice combined-an observation that builds confidence in the reproducibility of these findings.

The potential anatomic substrate for the hot spots was further identified by additional immunostaining of adjacent registered sections. Figure 5A shows an immunohistochemistry staining for in vivo-administered zalutumumab. The intratumoral zalutumumab distribution is patchier than the EGFr distribution and also different from the ${ }^{111}$ In distribution. Both stainings were negative in the necrotic regions and at the site of the hot spot. Examples of immunohistochemistry macrophage stainings are depicted in Figures $5 \mathrm{~B}$ and $5 \mathrm{C}$. In general, macrophages were located exclusively at the outer rim of the tumor. In the section shown in Figure 5B, we observed many macrophages at the site of the hot spot, suggesting that ${ }^{111}$ In accumulation may have occurred in these cells. However, this finding was not observed for all hot spots. Figure 5C shows a case in which macrophages were also found at the outer rim of the tumor, but without obvious colocalization with the hot spot.

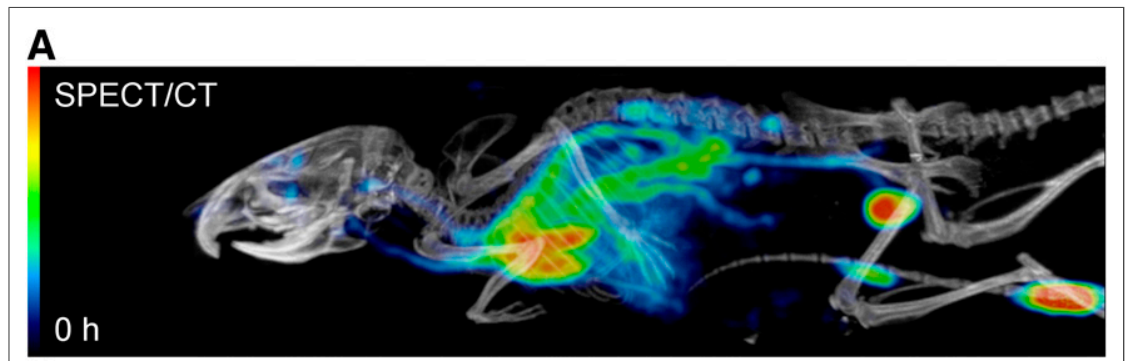

B

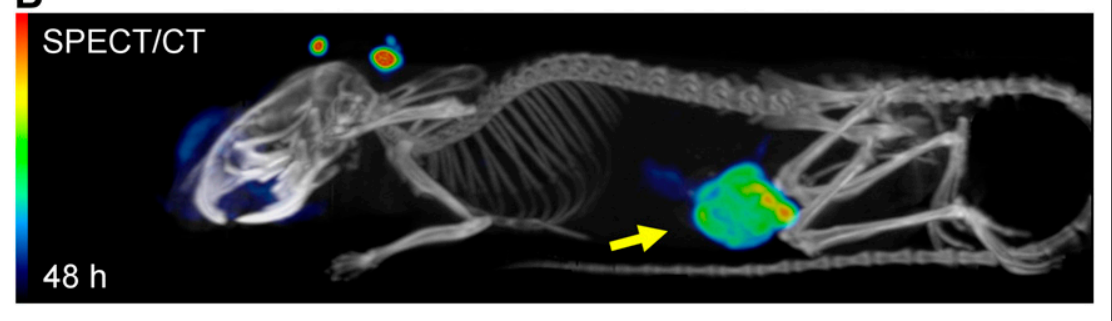

FIGURE 2. Total-body fused SPECT/CT maximum-intensity projections showing biodistribution of ${ }^{111} \mathrm{In}$-labeled zalutumumab directly after injection $(A)$ and $2 \mathrm{~d}$ after injection (B). Arrow points to A431 xenograft.

\section{DISCUSSION}

The results from this study show that within $48 \mathrm{~h},{ }^{111}$ In-labeled zalutumumab accumulated in a mouse A431 xenograft to such an extent that its in vivo intratumoral distribution could be noninvasively imaged at a high resolution using SPECT. The SPECT images reflect heterogeneous antibody uptake inside the tumor, a finding that was also encountered in studies using 2D autoradiography (31). By 3D coregistration of the focused SPECT tumor image with an entire stack of histologic sections, we found that the reconstructed radioactive tracer distribution showed many morphologic similarities to the EGFr target distribution and that the fraction of EGFr-negative area correlated well with the fraction of area with low ${ }^{111}$ In uptake. To our knowledge, this is the first time such analyses have successfully been performed at the level of individual SPECT tumor slices. 


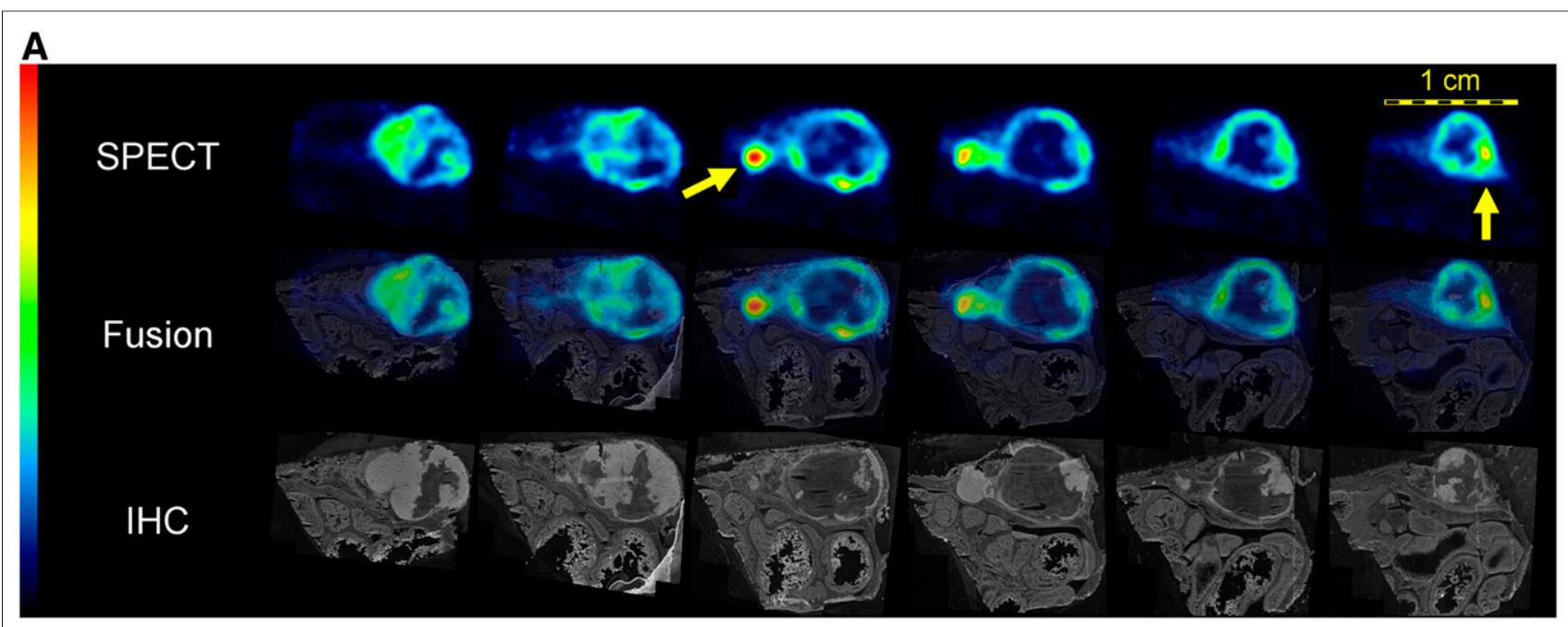

B

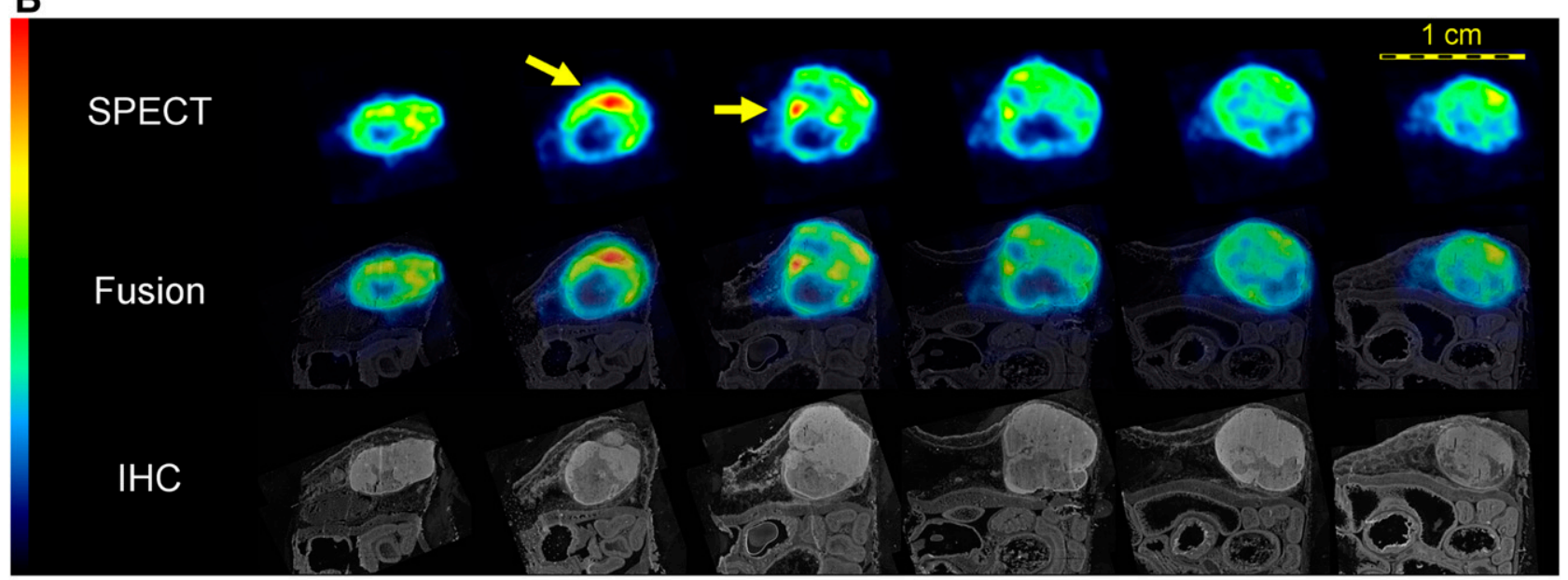

C

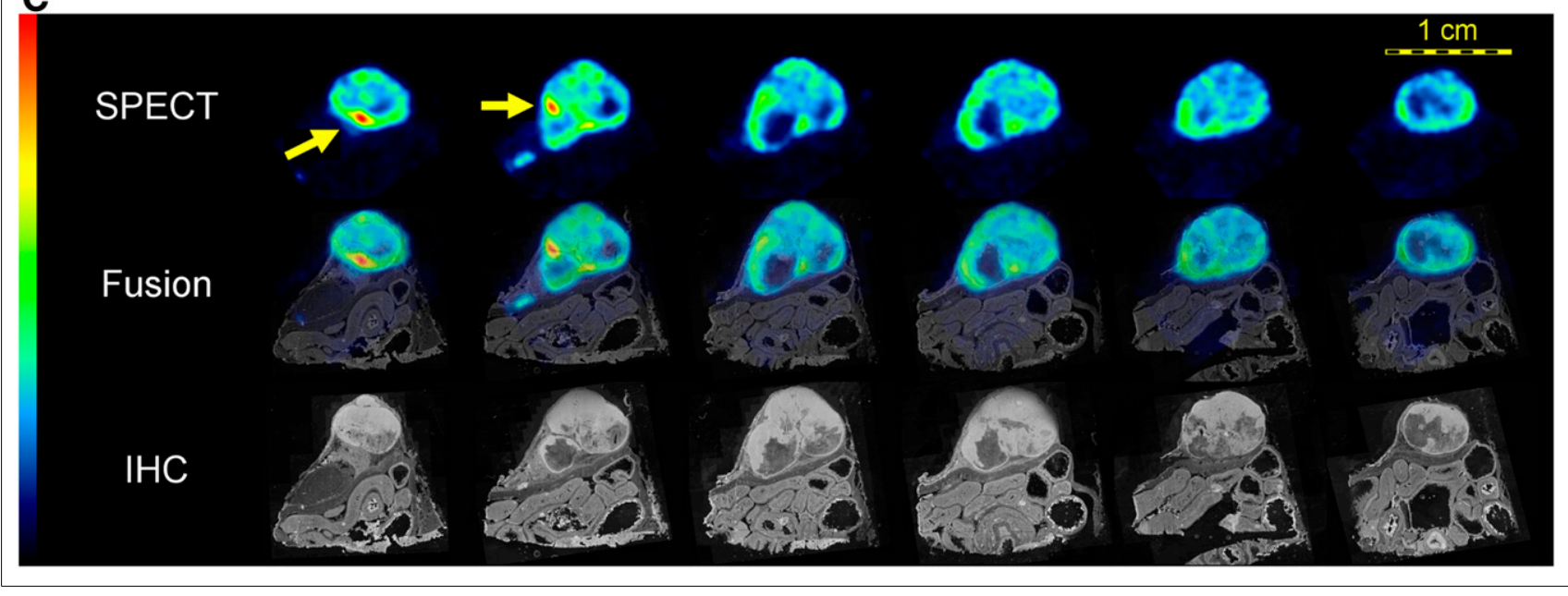

FIGURE 3. High-resolution in vivo SPECT slices of ${ }^{111} \mathrm{In}$ distribution in A431 xenograft compared with cross-sections through registered $3 \mathrm{D}$ immunohistochemistry (IHC) stack. Top rows: equidistant consecutive SPECT slices. Middle rows: SPECT images fused with registered immunohistochemistry slices stained for EGFr expression. Bottom rows: nonfused immunohistochemistry slices. Arrows point to hot spots that seem to be located at outer rim of or outside EGFr regions. Panels A, B, and C show results from mice 1, 2, and 3, respectively.

Tumor regions with virtual absence of EGFr expression were largely necrotic, as could be inferred from impaired cellular integrity observed in hematoxylin-eosin staining. This finding suggests that the low SPECT tracer content in certain parts is not due to poor antibody penetration into the tumor but to the lack of viable EGFr target-expressing tumor cells to which the antibody could bind. 


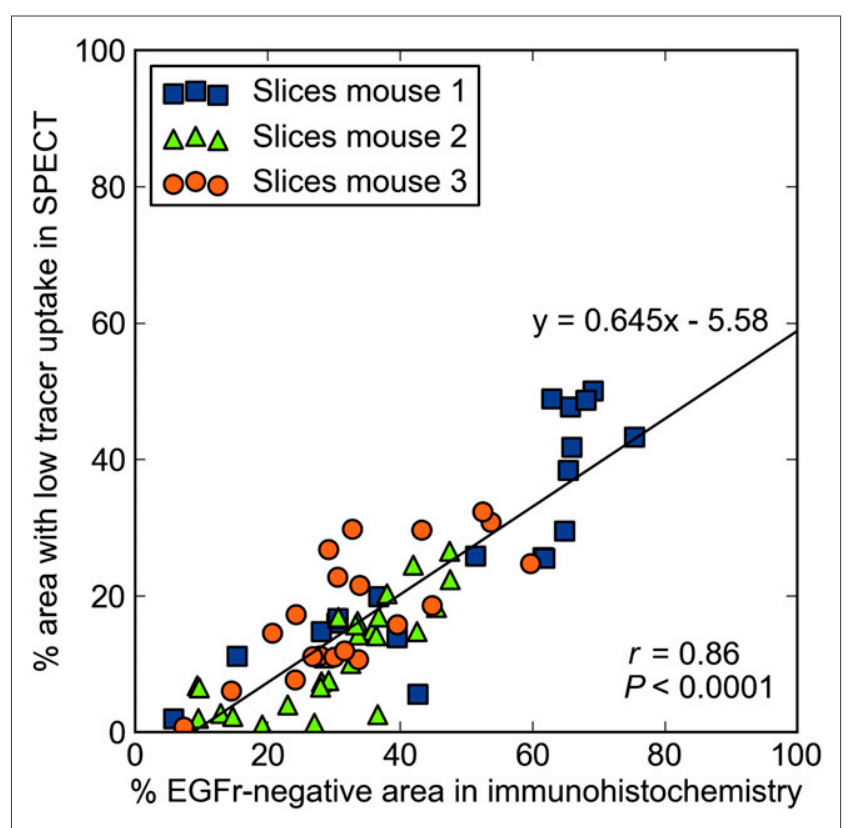

FIGURE 4. Scatterplot relating EGFr-negative (necrotic) tissue to regions with low SPECT uptake, both quantified as percentage of total slice area.

Good correlation coefficients were already found between EGFr-negative and SPECT-negative (low-uptake) areas in different slices by applying only simple thresholding. However, the method needs to be developed further to achieve reliable absolute

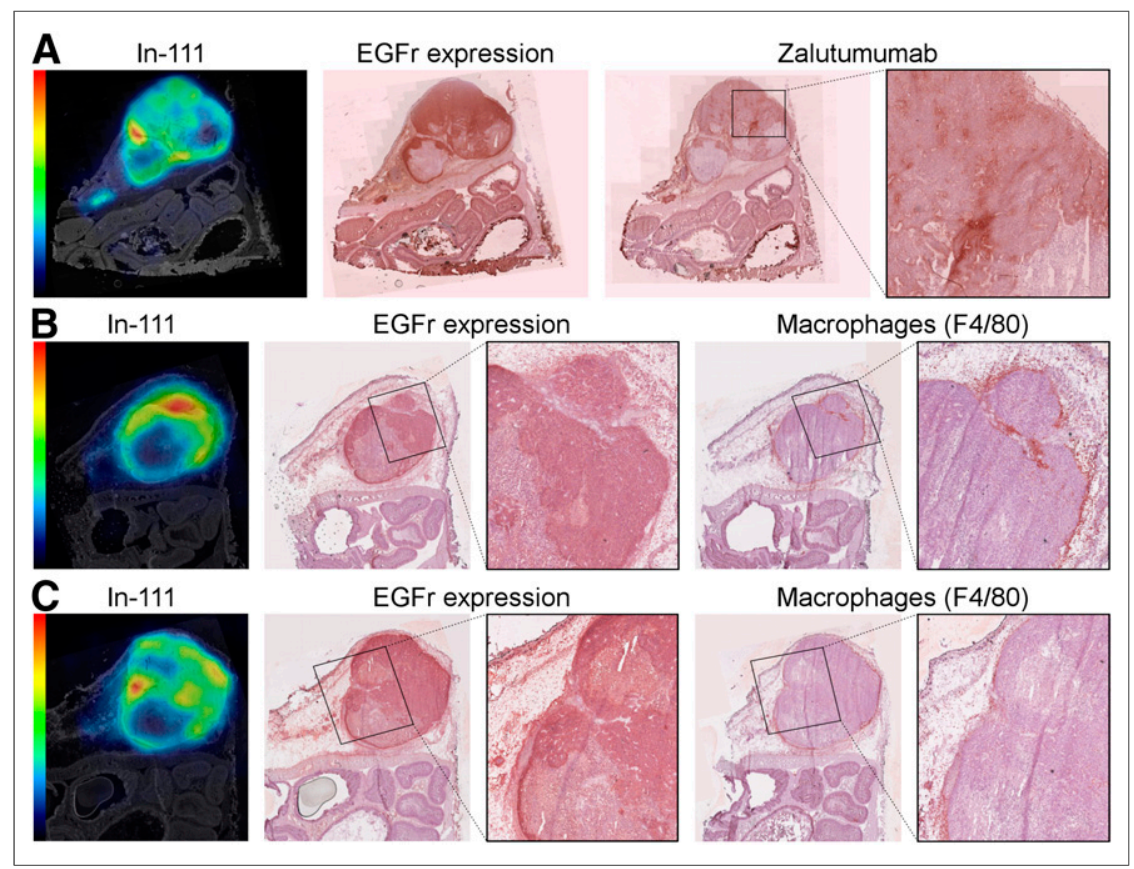

FIGURE 5. Expression and distribution of radiolabel ( ${ }^{111} \mathrm{In}$ ), EGFr, antibody (zalutumumab), and macrophages (F4/80). (A) SPECT slice from mouse 3 compared with 2 registered adjacent sections stained for EGFr expression (ex vivo incubation with zalutumumab) and presence of in vivo administered zalutumumab. Arrows point to ${ }^{111} \mathrm{In}$ hot spot in region with neither EGFr uptake nor zalutumumab expression. Magnification shows heterogeneous distribution of zalutumumab. (B) Similar example from mouse 2 in which ${ }^{111}$ In hot spot was found to coincide with EGFr-positive tumor tissue and macrophages. (C) Similar example from mouse 2, where arrow points to hot spot in EGFr-positive tumor area that does not express macrophages. quantitation of the necrotic area. For example, Figure 4 suggests that even for threshold values around the optimum with respect to correlation, simple thresholding of the SPECT volume may still underestimate the necrotic area by as much as $40 \%$. This underestimation appears to be systematic and most likely results from partial-volume effects due to the limited spatial resolution of the SPECT images. To improve the predictive value of SPECT for EGFr-negative tumor tissue, more advanced segmentation methods may be developed in the future, and newly developed SPECT systems with improved resolution, such as the recently launched U-SPECT + (32), may further reduce partial-volume effects. Nevertheless, the full 3D analysis of the 3 tumors in this study demonstrates that correspondence of low SPECT activity with necrosis can be reproduced over multiple entire tumor volumes with a simple algorithm. Although Figure 3 displays the full series of equidistant slices only in the transaxial direction, good correspondence was found also for crosssections in the other 2 orthogonal directions (Supplemental Figs. 1-3).

The applied method of coregistration maps many SPECT patterns onto morphologically similar EGFr patterns, but some ${ }^{111}$ In hot spots are mapped onto low-EGFr-expressing regions. It is unlikely that this discrepancy reflects registration error or resolution effects, for several reasons. First, the small hot spots in the SPECT images (arrows in Fig. 3) seem to be consistently mapped to morphologic indentations in the EGFr region borders. Second, injected zalutumumab (as determined by immunohistochemistry) was detected only in high-EGFr-expressing regions (Fig. 5A), whereas the ${ }^{111}$ In distribution completely covered all regions of EGFr expression. The disagreement between the SPECT and injected zalutumumab tissue distributions likely reflects separation of the ${ }^{111}$ In label from the zalutumumab and a difference in routing on internalization into tumor cells after target binding. Internalized zalutumumab (IgG) will be slowly degraded (9), preventing immunohistochemistry detection at $48 \mathrm{~h}$ after injection, whereas ${ }^{111} \mathrm{In}$ is expected to accumulate in the tumor cells since it is known to be a residualizing label $(33,34)$. Because we observed dense populations of macrophages in particular at the rim of the tumor, outside the EGFr area, it could be that some SPECT hot spots resulted from phagocytosed dead tumor cells containing residualized ${ }^{111} \mathrm{In}$. This possibility could explain the ${ }^{111}$ In accumulation outside the EGFrexpressing tumor mass shown by some of our data (Fig. 5). However, the fact that not all hot spots coincided with macrophage presence (Fig. 5C) indicates that other mechanisms also may contribute to the final arrangement of the ${ }^{111} \mathrm{In}$ at the time of the SPECT scan.

To investigate such mechanisms, future studies may also require the construction of a registered 3D stack of immunohistochemistry slices. Although the procedure described in this paper resulted in good registration between SPECT $\left({ }^{111}\right.$ In) and immunohistochemistry (EGFr target), the method is labor-intensive and depends on the presence of shared morphologic details between the optical photographs, the stained immunohistochemistry tissue sections, and the SPECT image. In 
addition, the application of only an affine transformation model requires the tissue to be fixated by careful snap-freezing, since chemical fixatives typically introduce substantial and not easily restored heterogeneous deformation of the tissue (35). Furthermore, our method of snap-freezing the entire animal had the practical disadvantage that the tumor could not be separately weighed and measured in a dose calibrator. More research is needed to devise a fully automatic method, which should not only be able to restore tissue processing deformations but also be robust to staining artifacts.

Radionuclide techniques have been successfully applied to discriminate between tumors with different levels of EGFr expression in vivo $(16,17,36-38)$, but some studies report discrepancies between EGFr expression and antibody binding $(37,39)$. Hoeben et al. (16) suggest a possible explanation by presenting evidence from 2D autoradiography showing that high-EGFr-expressing tumors may exhibit subcompartments with low EGFr expression. In this study, we replicated and validated these results in vivo and in 3 dimensions. An additional advantage of SPECT is that the heterogeneity of target receptor distributions and the process of IgG internalization can be monitored in the same animal over time, under non-target-saturating conditions. This 4-dimensional imaging capability may provide new insights into the encountered disparity between EGFr expression and antibody uptake in mouse tumors.

\section{CONCLUSION}

This study showed that sub-half-millimeter multipinhole SPECT noninvasively acquires high-resolution $3 \mathrm{D}$ in vivo images that allow clear visualization of the heterogeneous distribution of radioactive labeled antibodies in subcompartments of small tumors in mice. The reconstructed SPECT tracer distributions are morphologically similar to those of EGFr expression. However, some apparent differences between the distributions of tracer and EGFr accentuate the fact that the SPECT images show ${ }^{111} \mathrm{In}$ distribution, including both intact labeled antibody and free ${ }^{111} \mathrm{In}$ accumulated after antibody internalization and degradation. By taking into account the heterogeneity of distribution, ultra-highresolution SPECT permits a better estimation of antibody accumulation and target saturation in vital tumor parts. This ability may prove to be valuable in antibody dose optimization or in assessing the efficacy of antibody constructs. In summary, the focused multipinhole setup with high pinhole magnification provides researchers with a powerful tool to further optimize future anticancer medication.

\section{DISCLOSURE}

The costs of publication of this article were defrayed in part by the payment of page charges. Therefore, and solely to indicate this fact, this article is hereby marked "advertisement" in accordance with 18 USC section 1734 . No potential conflict of interest relevant to this article was reported.

\section{ACKNOWLEDGMENTS}

We thank Natalie ter Hoeve (UMC Utrecht) and Mark Begieneman (VUmc) for their help with tissue sectioning.

\section{REFERENCES}

1. Brindle K. New approaches for imaging tumour responses to treatment. Nat Rev Cancer. 2008;8:94-107.
2. Adams GP, Weiner LM. Monoclonal antibody therapy of cancer. Nat Biotechnol. 2005;23:1147-1157.

3. Van Dongen GAMS, Visser GWM, Lub-de Hooge MN, de Vries EG, Perk LR. Immuno-PET: a navigator in monoclonal antibody development and applications. Oncologist. 2007;12:1379-1389.

4. Wu AM, Senter PD. Arming antibodies: prospects and challenges for immunoconjugates. Nat Biotechnol. 2005;23:1137-1146.

5. Das M, Mohanty C, Sahoo SK. Ligand-based targeted therapy for cancer tissue. Expert Opin Drug Deliv. 2009;6:285-304.

6. Machiels J-P, Subramanian S, Ruzsa A, et al. Zalutumumab plus best supportive care versus best supportive care alone in patients with recurrent or metastatic squamous-cell carcinoma of the head and neck after failure of platinum-based chemotherapy: an open-label, randomised phase 3 trial. Lancet Oncol. 2011; 12:333-343.

7. Herbst RS. Review of epidermal growth factor receptor biology. Int J Radiat Oncol Biol Phys. 2004;59:21-26.

8. Rivera F, Vega-Villegas ME, Lopez-Brea MF, Marquez R. Current situation of panitumumab, matuzumab, nimotuzumab and zalutumumab. Acta Oncol. 2008; 47:9-19.

9. Lammerts van Bueren JJ, Bleeker WK, Bøgh HO, et al. Effect of target dynamics on pharmacokinetics of a novel therapeutic antibody against the epidermal growth factor receptor: implications for the mechanisms of action. Cancer Res. 2006;66:7630-7638.

10. Basu S, Kwee TC, Gatenby R, Saboury B, Torigian DA, Alavi A. Evolving role of molecular imaging with PET in detecting and characterizing heterogeneity of cancer tissue at the primary and metastatic sites, a plausible explanation for failed attempts to cure malignant disorders. Eur J Nucl Med Mol Imaging. 2011;38:987-991.

11. Goorden MC, van der Have F, Kreuger R, et al. VECTor: a preclinical imaging system for simultaneous submillimeter SPECT and PET. J Nucl Med. 2013;54:306312 .

12. Van der Have F, Vastenhouw B, Ramakers RM, et al. U-SPECT-II: an ultra-highresolution device for molecular small-animal imaging. J Nucl Med. 2009;50:599605.

13. Wu C, Have F, Vastenhouw B, Dierckx RAJO, Paans AMJ, Beekman FJ. Absolute quantitative total-body small-animal SPECT with focusing pinholes. Eur $J$ Nucl Med Mol Imaging. 2010;37:2127-2135.

14. Wu C, de Jong JR, Gratama van Andel HA, et al. Quantitative multi-pinhole small-animal SPECT: uniform versus non-uniform Chang attenuation correction. Phys Med Biol. 2011;56:N183-N193.

15. Chen C-L, Wang Y, Lee JJS, Tsui BMW. Toward quantitative small animal pinhole SPECT: assessment of quantitation accuracy prior to image compensations. Mol Imaging Biol. 2009;11:195-203.

16. Hoeben BAW, Molkenboer-Kuenen JDM, Oyen WJG, et al. Radiolabeled cetuximab: Dose optimization for epidermal growth factor receptor imaging in a headand-neck squamous cell carcinoma model. Int J Cancer. 2011;129:870-878.

17. Heskamp S, van Laarhoven HWM, Molkenboer-Kuenen JDM, et al. ImmunoSPECT and immunoPET of IGF-1R expression with the radiolabeled antibody R1507 in a triple-negative breast cancer model. J Nucl Med. 2010;51:1565-1572.

18. Penheiter AR, Griesmann GE, Federspiel MJ, Dingli D, Russell SJ, Carlson SK. Pinhole micro-SPECT/CT for noninvasive monitoring and quantitation of oncolytic virus dispersion and percent infection in solid tumors. Gene Ther. 2012;19:279-287.

19. Umeda IO, Tani K, Tsuda K, et al. High resolution SPECT imaging for visualization of intratumoral heterogeneity using a SPECT/CT scanner dedicated for small animal imaging. Ann Nucl Med. 2012;26:67-76.

20. Zhou Y, Shao G, Liu S. Monitoring breast tumor lung metastasis by U-SPECT-II/ CT with an integrin $\alpha_{v} \beta_{3}$-targeted radiotracer ${ }^{99 m}$ Tc-3P-RGD(2). Theranostics. 2012;2:577-588

21. Shao G, Zhou Y, Wang F, Liu S. Monitoring glioma growth and tumor necrosis with the U-SPECT-II/CT scanner by targeting integrin $\alpha \mathrm{v} \beta 3$. Mol Imaging. 2013;12:39-48.

22. Deleye S, Van Holen R, Verhaeghe J, Vandenberghe S, Stroobants S, Staelens S. Performance evaluation of small-animal multipinhole $\mu$ SPECT scanners for mouse imaging. Eur J Nucl Med Mol Imaging. 2013;40:744-758.

23. Bleeker WK, Lammerts van Bueren JJ, van Ojik HH, et al. Dual mode of action of a human anti-epidermal growth factor receptor monoclonal antibody for cancer therapy. J Immunol. 2004;173:4699-4707.

24. Perk LR, Visser GWM, Vosjan MJWD, et al. ${ }^{89} \mathrm{Zr}$ as a PET surrogate radioisotope for scouting biodistribution of the therapeutic radiometals ${ }^{90} \mathrm{Y}$ and ${ }^{177} \mathrm{Lu}$ in tumor-bearing nude mice after coupling to the internalizing antibody cetuximab. J Nucl Med. 2005;46:1898-1906.

25. Masui H, Moroyama T, Mendelsohn J. Mechanism of antitumor activity in mice for anti-epidermal growth factor receptor monoclonal antibodies with different isotypes. Cancer Res. 1986;46:5592-5598. 
26. Tomayko MM, Reynolds CP. Determination of subcutaneous tumor size in athymic (nude) mice. Cancer Chemother Pharmacol. 1989;24:148-154.

27. Branderhorst W, Vastenhouw B, van der Have F, Blezer ELA, Bleeker WK, Beekman FJ. Targeted multi-pinhole SPECT. Eur J Nucl Med Mol Imaging. 2011;38:552-561.

28. Branderhorst W, Vastenhouw B, Beekman FJ. Pixel-based subsets for rapid multi-pinhole SPECT reconstruction. Phys Med Biol. 2010;55:2023-2034.

29. Thévenaz P, Ruttimann UE, Unser M. A pyramid approach to subpixel registration based on intensity. IEEE Trans Image Process. 1998;7:27-41.

30. Klein S, Staring M, Murphy K, Viergever MA, Pluim JPW. Elastix: a toolbox for intensity-based medical image registration. IEEE Trans Med Imaging. 2010;29:196205.

31. Senekowitsch-Schmidtke R, Steiner K, Haunschild J, Möllenstädt S, Truckenbrodt R. In vivo evaluation of epidermal growth factor (EGF) receptor density on human tumor xenografts using radiolabeled EGF and anti-(EGF receptor) mAb 425. Cancer Immunol Immunother. 1996;42:108-114.

32. Ivashchenko O, van der Have F, Villena J, Beekman FJ. Quarter millimeter resolution pre-clinical SPECT with quarter-mm pinholes. Presented at: Annual Congress of the European Association of Nuclear Medicine, October 19-23, 2013, Lyon, France.
33. Mattes MJ, Griffiths GL, Diril H, Goldenberg DM, Ong GL, Shih LB. Processing of antibody-radioisotope conjugates after binding to the surface of tumor cells. Cancer. 1994;73:787-793.

34. Press OW, Shan D, Howell-Clark J, et al. Comparative metabolism and retention of iodine-125, yttrium-90, and indium-111 radioimmunoconjugates by cancer cells. Cancer Res. 1996;56:2123-2129.

35. Groen HC, van Walsum T, Rozie S, et al. Three-dimensional registration of histology of human atherosclerotic carotid plaques to in-vivo imaging. J Biomech. 2010;43:2087-2092.

36. Huang L, Gainkam LOT, Caveliers V, et al. SPECT imaging with ${ }^{99 \mathrm{~m}} \mathrm{Tc}$-labeled EGFR-specific nanobody for in vivo monitoring of EGFR expression. Mol Imaging Biol. 2008;10:167-175.

37. Aerts HJWL, Dubois L, Perk L, et al. Disparity between in vivo EGFR expression and ${ }^{89} \mathrm{Zr}$-labeled cetuximab uptake assessed with PET. J Nucl Med. 2009;50:123-131.

38. Cai W, Chen K, He L, Cao Q, Koong A, Chen X. Quantitative PET of EGFR expression in xenograft-bearing mice using ${ }^{64} \mathrm{Cu}$-labeled cetuximab, a chimeric anti-EGFR monoclonal antibody. Eur J Nucl Med Mol Imaging. 2007;34:850-858.

39. Niu G, Sun X, Cao Q, et al. Cetuximab-based immunotherapy and radioimmunotherapy of head and neck squamous cell carcinoma. Clin Cancer Res. 2010;16:2095-2105. 